\title{
Power Curve Characterization I: improving the bin method
}

\author{
A. Llombart ${ }^{1}$, S. J. Watson ${ }^{2}$, D. Llombart ${ }^{1}$ and J. M. Fandos \\ Department of Electrical Engineering \\ ${ }^{1}$ Fundacion CIRCE, University of Zaragoza \\ C/ Maria de Luna 3, 50018 Zaragoza (Spain) \\ Phone/Fax number:+34 976 762398, e-mail: llombart@unizar.es \\ ${ }^{2}$ Centre for Renewable Energy Systems Technology, Loughborough University, Ashby Road, Loughborough, \\ Leicestershire, LE11 3TU, UK \\ Phone: +44 1509 222421, Fax: +44 1509 610031, e-mail: s.j.watson@lboro.ac.uk
}

\begin{abstract}
Neural network approaches have been used to try and characterise the power performance of wind turbines in a wind farm. Stochastic techniques are often dismissed as being inferior to those which use neural networks but those stochastic techniques proposed are often overly simplistic. We propose modifications to the IEC $61400-12$ bin method in order to provide a more accurate characterisation of wind turbine performance in complex terrain taking account of direction and using a varying power law dependence of power output on wind speed. The method is validated using data from a real wind farm
\end{abstract}

\section{Key words}

Wind energy, power curve, bin method, power performance

\section{Introduction}

The cost effective operation and maintenance of wind farms requires accurate and informed condition monitoring of the wind turbines. One such technique is to analyse over time the power performance of the turbines.

For the latest wind turbines, e.g. $>600 \mathrm{~kW}$, there is limited data available to assess the long-term power performance of these machines. In addition, in Spain there are concerns in relation to the long-term operation in sites with highly complex terrain and high levels of turbulence intensity [1] - [4].

To assess the efficiency of a wind turbine the relationship between the wind mean speed (taken at the meteorological mast or at the nacelle of each wind turbine) and the power generated by each wind turbine must be found. But the best method to do this is not clear. There are some factors that complicate this relationship:

1. The time delay in the wind speed propagation and how this affects the correlation between mast and turbine wind speed;

2. The topography of the wind farm site;

3. The presence of nearby obstacles.
Moreover, the increasing size of wind farms makes the effect of these factors worse due to the increasing distance between some of the wind turbines and the meteorological mast.

Several studies have been carried out on this topic. In [1] a Neural Network (NN) with Kalman filter training is described. This work is improved in [2] where the authors achieved good results using NN. However, to achieve these results, they used data from two meteorological towers, and the furthest turbine was only $600 \mathrm{~m}$ from the nearest tower mast.

In [3], a method is presented that allows the detection of a decrease in the efficiency of any turbine up to a distance of $4 \mathrm{~km}$ from the meteorological mast. This method does not provide a power estimation, however, so it cannot be used for anything more than efficiency evaluation. In addition, this method takes six weeks to detect any problem with the turbine power performance.

Finally, in [4] it is suggested that the use of NNs is suited to the problem of power performance evaluation but the issue of distance from mast to turbine is not really addressed.

References [1], [2] and [4] are based solely on the use of Neural Networks. These references compare the use of NNs with relatively simple stochastic methods and conclude that the NN approach is superior, however, the stochastic methods chosen are overly simplistic.

In this paper, different stochastic methods that can be used to solve this problem are reviewed. The bin method is studied here in some detail, including that described in IEC 61400-12. However, we also consider a variation on this method with some extra modifications. Both the wind speed taken at the meteorological mast and that taken at the nacelle are used to make the power performance characterization of the turbine in order to compare the accuracy of the different methods. 
To show the value of the various modifications proposed, the results are tested with real data of a wind farm situated in Aragon in Spain.

\section{The stochastic methods}

The first stochastic method that has been used to infer the power of any wind turbine from the speed at the meteorological mast is to derive it from the manufacturer's power curve, possibly taking into account the correction due to the changes in air density. This method can also use the wind speed measured on the nacelle of each turbine.

The power curve given by the manufacturer has been obtained in ideal conditions, and clearly the turbines in an operational wind farms are not placed ideally in relation to the meteorological mast in the wind farm site. This means that the 'ideal' power curve is not always suitable for assessing the power performance of a wind turbine operating in a wind farm, particularly if the terrain is relatively complex.

To get a more accurate definition of the power curve for each turbine, this can be calculated from real historical data from the meteorological mast and the different turbines in the wind farm. There are some probabilistic methods to obtain the relationship between the mast and turbine:

- Bin method

- Regression

In this paper, we are going to deal with the bin method, which is outlined in the IEC 61400-12 standard. This consists of taking the mean values of the wind speed and the output power for a period of ten minutes. These values are then normalised to a reference air density. Once normalised the data are grouped using $0,5 \mathrm{~m} / \mathrm{s}$ bins (data recorded from directions that are influenced by obstacles, including the wind turbines and the meteorological masts, are excluded). The mean values of each bin are calculated as follows:

$$
\begin{gathered}
V_{i}=\frac{1}{N_{i}} \sum_{j=1}^{N i} V_{n, i, j} \\
P_{i}=\frac{1}{N_{i}} \sum_{j=1}^{N i} P_{n, i, j}
\end{gathered}
$$

where

$V_{i}$ is the normalised and averaged wind speed in bin $i$ $V_{n, i, j}$ is the normalised wind speed of data set $j$ in bin $i$ $P_{i}$ is the normalised and averaged power output in bin $i$ $P_{n, i, j}$ is the normalised power output of data set $j$ in bin $i$ $N_{i}$ is the number of 10 min data sets in bin $i$

The different values obtained for each bin define the relationship that is being determined.
Obviously, this method is valid to obtain the normalised power curve of a wind turbine, but, if this method is used to model the behaviour over time of the output power of the wind turbines in relation to the wind speed some questions arise immediately:

1. How should the sectors affected by obstacles be modelled?

2. Are the equations (1) and (2) above the most appropriate to calculate $V_{i}$ and $P_{i}$ ?

3. Why not use a method that takes into account the cubic relationship between wind speed and power? Or maybe some other relationship?

4. Why not use a model that considers the different behaviour of the wind turbines in different sectors rather than treating all sectors the same as dictated by the IEC 61400-12 standard?

\section{Methodology}

To answer these questions, an analysis has been undertaken using a year of historical data from a wind farm.

The wind farm chosen to perform the different tests is situated in Aragon (Spain). Due to confidentiality issues it is not allowed to present it in detail but, in order to understand the type of the terrain and to present the situation of the wind turbines whose data are used to undertake the study, a simplified map is presented (Fig. 1).

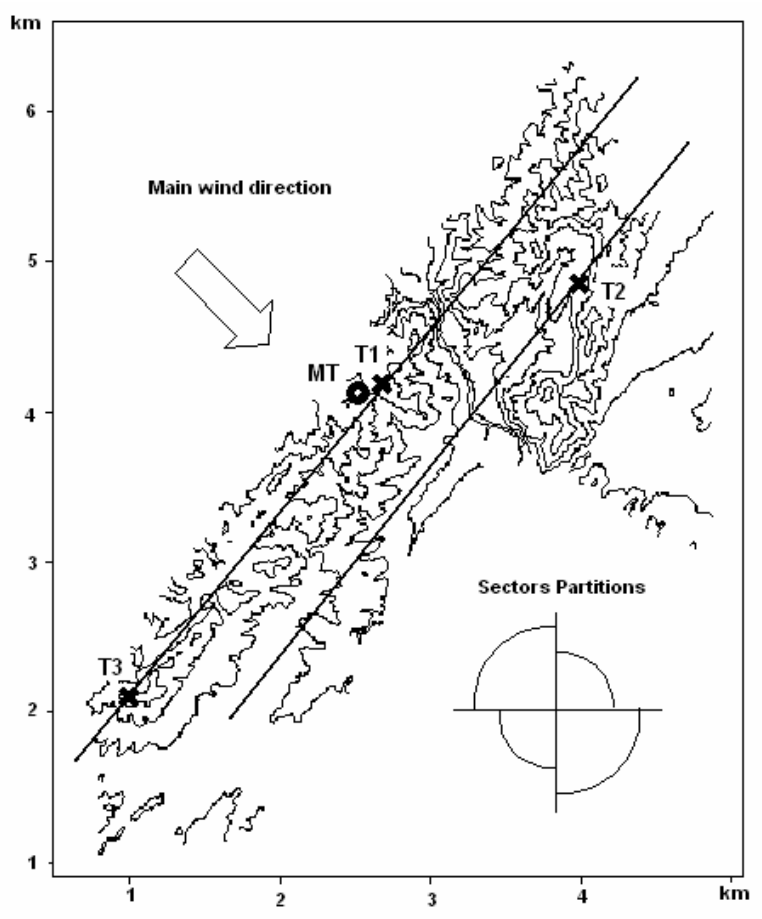

Figure 1.- A simplified view of the wind farm

In this map, it can be seen that the terrain is extremely complex, the turbines are disposed in two lines (following, more or less, the straight lines represented in 
the map) and the main wind direction is indicated by the big arrow in the top left corner.

The turbines considered, have been chosen taking into account their distance to the meteorological tower, turbine 1 is near of it, and turbine 2 and 3 are further. The objective of this election is to corroborate the influence of the distance in the characterization method. The main direction of the wind is also shown.

The analysis has been divided in three different tests:

- Bin method characterization.

- Bin method characterization with normalized power.

- Bin method characterization with normalized power and sector partition.

To compare the different results obtained it has been calculated the mean squared error of each approach, for the different wind turbines.

Following the different tests' that have been made are explained.

\section{A. Bin method characterization}

The bin method has been applied to each wind turbine as it is explained in paragraph 2, but, opposite to that, all the data have been taken as valid independently of their wind direction.

The power curve that characterizes the wind turbine behaviour is composed by the link of the different points obtained. So, the theoretical power that corresponds to each wind speed, can be obtained through the interpolation between the two nearest bins.

To obtain the mean squared error it has been calculated the error between the theoretical and the real power for all the data in the full year.

\section{B. Bin method characterization with normalized power}

It is clear that the relationship between wind speed and power output, is not linear. If the bin characterised by wind speeds between 5 and 5,5 is observed, and a cubic relationship between the different variables is assumed, the theoretical power output associated with the wind speed at the maximum value of the bin is 33,1\% higher than that associated with the wind speed at the minimum value of the bin.

To take into account the "non linear" relationship between the wind speed and the power, a new method of obtaining the $V_{i}$ and $P_{i}$ values is proposed:

$$
V_{i}=\frac{V_{i, H}-V_{i, L}}{2}
$$

$$
P_{i}=\frac{1}{N_{i}} \sum_{j=1}^{N i} \frac{P_{n, i, j} \cdot V_{n, i, j}{ }^{k}}{V_{i}^{k}}
$$

where

$V_{i, H}$ is the maximum value of wind speed in bin $i$

$V_{i, L}$ is the minimum value of wind speed in bin $i$

$k$ is a factor that must be adjusted for each direction sector and each wind speed bin

To get the theoretical power that corresponds to any wind speed $(V)$ the following steps must be followed:

1) Select the bin that corresponds to the wind speed chosen.

2) Apply the following equation, in order to consider the relationship shown in eq. 2

$$
P_{v}=\frac{P_{i} \cdot V^{k}}{V_{i}^{k}}
$$

where

$P_{v}$ is the theoretical power corresponding to $V$

$V_{i}$ is the normalised and averaged wind speed in bin $i$

$P_{i}$ is the normalised and averaged power output in bin $i$

To get the characterization some $k$ values have been tested from 0,5 to 3 , in steps of 0,5 . Each bin have been characterized for the $k$ value that optimises the mean squared error, obviously the data taken to calculate the error are only those that belongs to this bin.

Finally, the mean squared error for the whole year has been calculated.

\section{Bin method characterization with sector partition}

We have carried out studies to analyse the effect of wind direction in the characterisation process. The data in each bin are grouped as a function of the direction and then $V_{i}$ and $P_{i}$ are calculated, for each sector.

The approach that we have been considered is a partition in 4 sectors. Taking as reference the direction in which the turbines are aligned, we have considered 4 sectors as it is shown in figure 1 (the sectors are defined as indicated in the bottom right corner).

This method has been used in combination with the method shown in paragraph B.

Each sector has been analysed separately and then the mean squared error for the whole year has been calculated.

The different characterization methods exposed can be applied using either the wind speed at the meteorological mast or that at the nacelle of each wind turbine. Obviously if the wind direction must be considered the only data available are those at the meteorological tower, 
that have been used independently of the wind speed considered.

\section{Results}

Before any characterization test the quality of the data must be checked. There are some situations that make that the data were not representative of the behaviour of the turbines. For example, the turbine may not work during part of the ten minutes time of a data set, so the power registered is not useful for the study, or some error in the communication/store/collection process may occur.

The different data used for this study have been filtered previously to avoid the consideration of any wrong data, and a set of right data have been obtained for each turbine and the meteorological tower.

The different methods explained in the paragraph 3 have been applied to the three turbines using both the wind speed taken at the meteorological mast and the wind speed taken in the nacelle of each turbine.

The following tables show the results obtained in the different approaches. There is a table for each wind turbine and for each wind speed used, in this tables the results of each method are shown. The mean squared error is presented and, for the different modifications proposed, the improvement measure as a percentage of the first method is presented too.

Firstly, the tables corresponding to the use of the wind speed at the meteorological mast are presented.

TABLE I. - Characterization results for turbine 1

\begin{tabular}{|l|c|c|}
\hline Turbine 1 & MSE & Improv. (\%) \\
\hline Method A & 1359,08 & \\
\hline Method B & 1357,48 & $0,12 \%$ \\
\hline Method C & 1217,59 & $10,41 \%$ \\
\hline
\end{tabular}

TABLE II. - Characterization results for turbine 2

\begin{tabular}{|l|c|c|}
\hline Turbine 2 & MSE & Improv. (\%) \\
\hline Method A & 2730,49 & \\
\hline Method B & 2717,29 & $0,48 \%$ \\
\hline Method C & 1837,47 & $32,71 \%$ \\
\hline
\end{tabular}

TABLE III. - Characterization results for turbine 3

\begin{tabular}{|l|c|c|}
\hline Turbine 3 & MSE & Improv. (\%) \\
\hline Method A & 5499,81 & \\
\hline Method B & 5471,83 & $0,51 \%$ \\
\hline Method C & 4507,16 & $18,05 \%$ \\
\hline
\end{tabular}

From the different results showed in the tables I to III it can be derived that:

- $\quad$ The method B improves the results slitghly

- The effectiveness of method $\mathrm{C}$ depends on the turbine position in relation to the meteorological mast. If the turbine is near of the meteorological mast the wind speed considered represents accurately the wind speed at the turbine, so the method cannot improve the results considerably.

- In spite of the improvement achieved by the modified methods, in those cases in which the turbine is far from the MT, the whole characterization is much worse than the characterization for the turbine near the MT.

Finally, the tables corresponding to the use of the wind speed at the nacelle of each wind turbine are presented. It must be remembered that when the data of wind direction are required they must be taken from the meteorological mast. For this case, the modification B doesn't give any advantage, so the results are not showed. Due to this, the method $\mathrm{C}$ has been carried out alone, not in combination with method B.

TABLE IV. - Characterization results for turbine 1

\begin{tabular}{|l|c|c|}
\hline Turbine 1 & MSE & Improv. (\%) \\
\hline Method A & 81,74 & \\
\hline Method C & 77,23 & $5,52 \%$ \\
\hline
\end{tabular}

TABLE V. - Characterization results for turbine 2

\begin{tabular}{|l|c|c|}
\hline Turbine 2 & MSE & Improv. (\%) \\
\hline Method A & 119,10 & \\
\hline Method C & 93,89 & $21,17 \%$ \\
\hline
\end{tabular}

TABLE VI. - Characterization results for turbine 3

\begin{tabular}{|l|c|c|}
\hline Turbine 3 & MSE & Improv. (\%) \\
\hline Method A & 137,35 & \\
\hline Method C & 125,90 & $8,33 \%$ \\
\hline
\end{tabular}

It can be observed that, for the wind turbines studied the use of the wind speed taken at nacelle is more suitable for the characterization of the wind turbines output power.

\section{Conclusion}

Characterisation of the wind turbine power curves in relation to the wind speed recorded at a meteorological mast that may be situated some distance from the turbine is a challenging problem that must be solved in order to optimise the operation and maintenance of the wind farm.

Some methods have been developed to deal with this issue based on Neural Network techniques. These methods do not consider large distances between turbines and the meteorological mast.

Stochastic techniques can be used but the error can be quite large if the statistical technique is overly simplistic. In order to use the bin method proposed in IEC-61400-12 in an operational wind farm in complex terrain, some changes are proposed which significantly reduce (between 13\% and 33\%) this error and take into account the distance between mast and turbine. 
To generalize the results for any wind farm is difficult but the results obtained show that the wind speed at the nacelle is more suitable for characterization purposes.

\section{Acknowledgement}

This work has been carried out with funding from the Spanish Ministerio de Ciencia y Tecnologia (projects: DPI2003-09731 and FIT-120000-2004-182) and the Europa XXI Program (CAI).

\section{References}

[1] S. Li, D. C. Wunsch, E. A. O’Hair and M. G. Giesselmann, "Wind turbine power estimation by neural networks with kalman filter training on a SIMD parallel machine”, International Joint
Conference on Neural Networks, Vol. 5, pp 3430 3434, July 1999

[2] S. Li, D. C. Wunsch, E. A. O’Hair and M. G. Giesselmann, "Using Neural Networks to Estimate Wind Turbine Power Generation", IEEE trans. On Energy Conversion, Vol. 16, $\mathrm{N}^{\mathrm{o}}$ 3, pp 276-282, September 2001

[3] A. Llombart, F. J. Val, M. Sanz, G. Costales, I. Gomez, "Time-continuous control system for supervision of the electrical power production of wind turbines"; European Wind Energy Conference, May 2003

[4] S. Kélouwani, K. Agbossou, "Nonlinear Model Identification of Wind Turbine with a Neural Network”, IEEE trans. On Energy Conversion, Vol. 19, No 3, pp 607-612, September 2004 